\title{
A Numerical Study of Laminar Natural Convection Heat Transfer and Radiation from a Rectangular Vertical Fin Array - Quasi-3D approach
}

\author{
Dr. P.V.J Mohan Rao \\ Professor \& Head of Marine Engineering Department A.U College of Engineering (Autonomous) \\ Andhra University, Visakhapatnam-530003 Andhra Pradesh, India.
}

\begin{abstract}
The problem of natural convection heat transfer from a vertical fin array is theoretically formulated by treating the adjacent internal fins as two fin enclosures. Three dimensional governing equations of mass, momentum and energy for the fluid in the two-fin enclosure together with the neat conduction equations in the fins are numerically solved using ADI method. The heat lost to the ambient fluid from the two end fins of the fin array is also computed separately. Heat transfer by radiation is also considered in the analysis. The numerical results are compared with the experimental data available in literature showing good agreement. The effects of system parameters such as the base temperature, fin height, fin spacing are studied on heat transfer rate and effectiveness of the vertical fin array. Results show that fin spacing is most significant parameter and there exists an optimum value for the fin spacing for which the heat transfer rate from the fin array is maximized. Results also show that higher heat transfer rates are obtained with vertical fin array of the same geometry. Study is limited to laminar convection only.
\end{abstract}

\section{NOMENCLATURE}

$A_{B}$ area of the horizontalbase plate, $(B W), m^{2}$ $A_{R}$ aspect ratio for two - fin enclosure, $(L / S)$

$A_{W}$ half the cross -

sectional area of the fin, $\left(t_{F} W\right), m^{2}$

$B$ breadth of the horizontal base plate, $m$

$C_{P}$ specific heat, $\mathrm{J} \mathrm{kg}^{-1} \mathrm{~K}^{-1}$

E Effectivenes

$F$ radiation shape factor

$g$ acceraltion due to gravity, $\mathrm{m} \mathrm{s}^{-2}$

$G_{r}$ Grashof number, $g \beta\left(T_{w, 0}-T_{\infty}\right) S^{3} / v_{f}^{2}$

$h$ heat transfer coefficent, $W \mathrm{~m}^{-2} \mathrm{~K}^{-1}$

$H$ height of the fin, $m$.

J radiosity, $\mathrm{Wm}^{-2}$

$k$ thermal conductivity, $W m^{-1} K^{-1}$

$L$ length of $f$ in, $m$

$N$ number of fins in the fin array

$p$ pressure, Pascals

$P$ half - perimeter of the fin, $\left(2 t_{F}+W\right), m$

Pr Prandtl number, ${ }^{c_{p f}} \mu_{f} / k_{f}$

$Q$ heat transfer rate, $W$

Ra Rayleigh number, $g \beta\left(T_{w, 0}-T_{\infty}\right) S^{3} /\left(v_{f} \alpha_{W}\right)$

$S$ spacing between adjacent fins, $m$

$t_{F}$ half - thickness of the fin, $m$

t time, $s$

$z$ position coordinate normal to the fin measured from the right fin, $m$ $\alpha$ thermal diffusivity of fluid, $(k / \rho c)_{f} m^{2} / s$

$\beta$ thermal expansion coefficient of fluid, $K^{-1}$ $\varepsilon$ emissivity

$\gamma$ temperature ratio parameter, $\left(T_{w, 0}-T_{\infty}\right) /$ $T_{\infty}$

$\mu$ dynamic viscosity, $\mathrm{kg} \mathrm{m}^{-1} \mathrm{~s}^{-1}$

$v$ kinematic velocity, $m^{2} s^{-1}$

$\rho$ density, $\mathrm{kg} \mathrm{m}^{-3}$

$\Psi$ stream function

$\sigma$ Stefan - Boltzman constant, $(5.67 \times$

$10^{-8} \mathrm{~W} \mathrm{~m}^{-2} \mathrm{~K}^{-4}$ )

$\theta_{W} \quad T_{W} / T_{\infty}$

$\zeta$ vorticity function, $s^{-1}$

\section{Subscripts}

1 fin a two-fin enclosure

3 base in a two-fin enclosure

5 end fin defined in Eq. (26a)

$\mathrm{B}$ base of the fin array

$\mathrm{B}, 0$ base plate in the absence of fins

$\mathrm{E}$ end fins of the fin array

I internal fins of the fin array

c convection

$\mathrm{f}$ fluid

$\mathrm{m}$ average

$r$ radiation

$T$ temperature, $K$

$u$ velocity component in $x$-direction, $m s^{-1}$

$v$ velocity component in $y-$ direction, $m s^{-1}$

$W$ width of the fin (also that of base plate), $m$

$\mathrm{T}$ total

W fin surface

w, 0 base of the fin

$x$ position coordinate along the fin measured from the base bifnthegdium

$y$ position coordinate along the fin height, $m$ 


\section{INTRODUCTION}

Extended surfaces, which are popularly known as fins, are extensively used in air-cooled automobile engines and in-cooled aircraft engines. Fins are used for the cooling of computer processors, and other electronic devices. There has been continuous research on improving the efficiency of heat exchange systems using fins involving natural convection and forced convection. Early experimental work on free convection in horizontal and vertical fin arrays was conducted by Starner and McManus [1], Jones and Smith [2]. Radiation heat transfer pays a important role in heat transfer from fin arrays. Manzoor et al. [3] analyzed the heat lost from the fins by convection and radiation through one dimensional and $2 \mathrm{~d}$ approach. Rammohan Rao and Venkateshan [4] carried our experiments on horizontal fin arrays. They showed that much larger heat fluxes are in short fins than long fins. Further the convective heat transfer increased linearly with fin spacing, while the increase in radiation heat transfer showed non-linear trend. M. Mobedi and H. Yuncu [5] numerically investigated natural convection heat transfer in a longitudinal short rectangular fin array on a horizontal base. They analysed flow configurations occurring in the channel of the fin arrays with different parameters like fin height, fin length and fin spacing. Yuncu and Anbar [6] conducted experiments by mounting different numbers of fins on a heated horizontal base plate of width $250 \mathrm{~mm}$. The fin spacing decreased as the number of fins was increased. They found that for a given base-to- ambient temperature difference the convection heat transfer rate from arrays reaches a maximum at a particular fin spacing and fin height. Guvenc and Yüncü [7] carried out an experimental investigation on performance of fin array and found that higher heat transfer enhancement is obtained with vertically oriented base than with horizontally oriented base for fin arrays of same geometry. Sane and Sukhatme [8] employed a quasi 3D approach by neglecting the velocity component along fin spacing for analyzing the problem of natural convection heat transfer from a horizontal rectangular fin array. Rao et al. [9] developed a numerical model treating the adjacent internal fins as two-fin enclosures for study the heat transfer from horizontal fin array. They studied the effects of system parameters like fin height, fin spacing, base temperature and emissivity on rate of heat transfer from fin array. Regression equations are also developed to readily calculate the average Nusselt number, heat transfer rate and effectiveness for a fin array.

A survey of literature reveals only a few theoretical studies on vertical fin arrays. The computational domain in their paper includes a single fin and half of the fin spacing (i.e., S/2), thus precluding the adjacent fin or fins. The experimental study of Yuncu and Anbar [6] indicates the effect of the adjacent fins on heat transfer. These observations prompted the authors to consider the heat transfer to the fluid in a limited enclosure from adjacent fin in a fin array, and also to include radiation mode of heat transfer in their analysis.

\section{PHYSICAL MODEL AND FORMULATION}

The two adjacent internal fins having a common base are shown in the figure. 1. The base is maintained at a constant temperature $T_{W, 0}$, and $T_{W, 0},>T_{\infty}$, where $T_{\infty}$ is ambient air temperature. The space between the fins is $\mathrm{S}$ in $\mathrm{Z}$ - direction. The length 1 and the height $\mathrm{H}$ of each fin are in $\mathrm{X}$-direction and $\mathrm{Y}$-direction respectively. The half-thickness of the fin is $t_{F}$. Heat transferred from the fins and the base to the ambient air by convection and radiation.

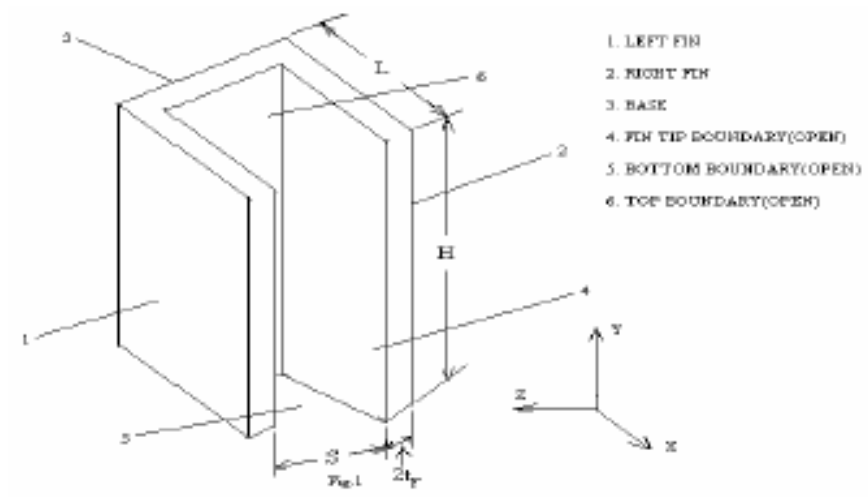

Fig.1 Physical model and configuration

The problem is formulated considering the two vertical fins and the vertical base together as a threedimensional enclosure. The two fins are the left, right and vertical base, top open, bottom open, fin top boundary open are boundaries of the enclosure are governed by the mass, momentum and energy balance equations for the fluid in conjugation with the two-dimensional heat conduction equation for each fin, which are given below. The cross flow velocity component, $\mathrm{w}$ in $\mathrm{Z}$ - direction is assumed to be zero (Sane and Sukhatme [8]). The 
radiation components of heat transfer from the fins and the base together are incorporated as the heat generation term in the energy balance equation for the fluid in the enclosure. Thus the equations considered are as follows.

Fluid medium

$\frac{\partial u}{\partial x}+\frac{\partial v}{\partial y}=0$

$\rho_{f}\left(\frac{\partial u}{\partial t}+u \frac{\partial u}{\partial x}+v \frac{\partial u}{\partial y}\right)=-\frac{\partial p}{\partial x}+\mu_{f}\left(\frac{\partial^{2} u}{\partial x^{2}}+\frac{\partial^{2} u}{\partial y^{2}}+\frac{\partial^{2} u}{\partial z^{2}}\right)$

$\rho_{f}\left(\frac{\partial v}{\partial t}+u \frac{\partial v}{\partial x}+v \frac{\partial v}{\partial y}\right)=$

$-\frac{\partial p}{\partial y}-\rho_{f}\left[1-\beta\left(T-T_{\infty}\right)\right]+\mu_{f}\left(\frac{\partial^{2} v}{\partial x^{2}}+\frac{\partial^{2} v}{\partial y^{2}}+\frac{\partial^{2} v}{\partial z^{2}}\right)$

$\frac{\partial p}{\partial z}=0$

$\rho_{f} C_{p f}\left(\frac{\partial T}{\partial t}+u \frac{\partial T}{\partial x}+\frac{\partial T}{\partial y}\right)=k_{f}\left(\frac{\partial^{2} T}{\partial x^{2}}+\frac{\partial^{2} T}{\partial y^{2}}+\frac{\partial^{2} T}{\partial z^{2}}\right)+q^{\prime \prime \prime}$

Where, $q^{\prime \prime \prime}=\left(2 H L q_{r 1}+S H q_{r 3}\right) /(S H L)$

Where $q_{r 1}=\left(E_{b 1}-J_{1}\right) /_{R_{1}}$ and $q_{r 3}=\left(E_{b 3}-J_{3}\right) /_{R_{3}}$ (Rao et. al. , [9])

Heat conduction in right fin

$\rho_{w} C_{p w} \frac{\partial T_{w}}{\partial t}=k_{w}\left(\frac{\partial^{2} T_{w}}{\partial x^{2}}+\frac{\partial^{2} T_{w}}{\partial y^{2}}\right)+\left.\frac{P}{A_{w}} k_{f} \frac{\partial T}{\partial z}\right|_{z=0}$

Heat conduction in right fin

$\rho_{w} C_{p w} \frac{\partial T_{w}}{\partial t}=k_{w}\left(\frac{\partial^{2} T_{w}}{\partial x^{2}}+\frac{\partial^{2} T_{w}}{\partial y^{2}}\right)+\left.\frac{P}{A_{w}} k_{f} \frac{\partial T}{\partial z}\right|_{z=S}$

Where $\mathrm{P}$ and $A_{w}$ are the half perimeter and half cross-sectional area of the fin respectively.

$P=2 t_{F}+H$ and $A_{w}=t_{f} H$

The u- and v- momentum balance equations, i.e., Eqs. (2) and (3) are coupled making use of vorticity $\zeta$, which is defined as follows.

$?=\frac{\partial v}{\partial x}+\frac{\partial u}{\partial y}$

The terms in Eq. (2) are differentiated with respect to y and those in Eq. (3) are differentiated with respect to $\mathrm{x}$. The resulting equations are subtracted one from the other to yield the following vorticity equation.

$?_{f}\left(\frac{\partial ?}{\partial t}+u \frac{\partial ?}{\partial x}+v \frac{\partial ?}{\partial y}\right)=?_{f} g \beta \frac{\partial T}{\partial x}+\mu_{f}\left(\frac{\partial^{2} ?}{\partial x^{2}}+\frac{\partial^{2} ?}{\partial y^{2}}+\frac{\partial^{2} ?}{\partial z^{2}}\right)$

Thus the problem is governed by the energy balance and vorticity equations, i.e, Eqs. (5) and (9), and the heat conduction equations for fins, i.e., Eqs. (6) and (7).

The stream function $\psi$ is defined as

$u=-\frac{\partial ?}{\partial y}, v=\frac{\partial ?}{\partial x}$

The vorticity equation in terms of stream function is as follows:

$\zeta=\frac{\partial^{2} ?}{\partial x^{2}}+\frac{\partial^{2} ?}{\partial y^{2}}$

The stream functions $\psi$ can be evaluated using Eq. (11) if the vorticities $\zeta$ are known. The velocity components $\mathrm{u}$ and $\mathrm{v}$ are to be computed from the values of $\psi$ through equation (10).

The boundary conditions at the left, right, base, Fin tip, bottom and top boundaries of the enclosure are given below:

Left boundary $(\mathrm{z}=0)$ :

$T=T_{w, 0}=$ constant for $x=0$ and $0 \leq y \leq H$ (fin base $)$;

$\frac{\partial T}{\partial x}=0$ at $x=L$ and $0 \leq y \leq H$ (fin tip);

$u=v=?=0,\left.?\right|_{z=0}=\frac{\partial \zeta}{\partial z}=0$ for $0 \leq x \leq L$ and $0 \leq y \leq H$

In the same fashion Right, Base, Fin tip, Top and Bottom boundaries are also defined but not mentioned here to conserve the space.

The values of vorticity $\zeta$ at the six boundaries are derived making use of relevant boundary conditions following Roache [10] and Mobedi and Yuncu [5].

All the above equations are normalized making use of the dimensionless variables.

\section{Heat transfer rate from a fin array, $Q_{T}$}

The rate of heat transfer from the left or right fin $Q_{1}$, base $Q_{3}$ and end fin $Q_{5}$ are calculated by using the related equations.

The breadth of the vertical base plate, on which the fins are horizontally mounted, is given B 


$$
B=(N-1) S+2 N t_{F}
$$

Where $\mathrm{B}$ is the breadth of the plate and $\mathrm{N}$ is the number of fins.

The heat transfer rates from the inner fins of the fin array $\left(Q_{I}\right)$, the baser of the fin array $\left(Q_{B}\right)$ and the two end fins $\left(Q_{E}\right)$ are given by

$Q_{I}=2(N-2) Q 1, Q B=Q_{3}, Q_{E}=2 Q_{5}$

$Q_{T}$, the total heat transfer rate from the fin array is given by

$Q_{T}=Q_{I}+Q_{B}+Q_{E}$

Fin array - average Nusselt number, $\mathbf{n} u_{m}$ and effectiveness, $\mathrm{E}$

Total heat transfer area for the $\mathrm{N}$-fin array is given by

$A_{T}=\left(B-2 N t_{F}\right)+2 N L\left(H+2 t_{F}\right)$

The average heat transfer coefficient, $h_{m}$ for the $\mathrm{N}$-fin array for combined convection and radiation is defined by the following equation

$h_{m}=\frac{Q_{T}}{A_{T}\left(T_{w, 0}-T_{\infty}\right)}$

The average Nusselt number for the $\mathrm{N}$-fin array is defined as

$h_{m}=h_{m} S / k_{f}$

$Q_{B, 0}$, the heat transfer rate from the vertical plate by convection and radiation is

$Q_{B, 0}=A_{B}\left[A_{T}\left(T_{w, 0}-T_{\infty}\right)+\epsilon_{3} \sigma\left(T_{w, 0}^{4}-T_{\infty}^{4}\right)\right]$

Where the $h_{B, 0}$ is calculated by using the equation which is available in literature (Guvenc and Yuncu [7])

The effectiveness of the fin array, $E=\frac{Q_{T}}{Q_{B, 0}}$

\section{METHOD OF SELECTION}

The procedure used to obtain the temperature and velocity fields as follows. The temperature fields in the two-fin enclosure and on the fins are obtained by the solution of the normalized energy balance equation for the fluid in conjugation with the heat conduction equations for fins, Alternating Direction Implicit (ADI) method (Roache [10]) is used to solve the energy balance equation for the fluid. The solution of these equations yields $T^{+}$and $\zeta^{+}$fields at all grid points in the enclosure at each step, $\Delta t^{+}$. Making use of $\zeta^{+}$, normalized stream functions $\psi^{+}$are obtained by Successive Over Relaxation (SOR) method. The normalized velocity components $u^{+}$and $v^{+}$at all $x^{+}$and $y^{+}$(i.e., at all gird points) are calculated. The procedure is continued for successive time steps until the steady temperature and velocity fields are obtained at all grid points in the twofin enclosure.

\section{RESULTS AND DISCUSSION}

Numerical results are obtained for a vertical eight fin array for two fin lengths, viz., $\quad L=15$ and 25 $\mathrm{mm}$ mounted on a fixed base of $250 \mathrm{~mm}$. These results are shown plotted in Fig.2 along with the experimental data of Guvenc and Yuncu [7]. A good agreement is observed between the numerical results and the experimental data. An increase in the convection heat transfer rate is observed with an increase in either base temperature, $T_{w, 0}$ or length of the fin, L. Fig. 3 presents the average Nusselt number as a function of Rayleigh number for two different fin lengths and two different fin emissivities. The numerical results are shown in Fig.3 for two different emissivities, namely $\varepsilon_{1}=0.15$ and $\varepsilon_{1}=0.85$ for fin length $L=25 \mathrm{~mm}$. As it can be expected, the Nusselt number is found to be more at higher fin emissivity and also it increases as Rayleigh number increases. An important outcome of the investigation is presented in Fig. 4, namely the effect of the fin spacing on heat transfer rate in a fin array. Numerical results are obtained for a number of fin spacings (S) ranging from 4 through $32 \mathrm{~mm}$. Results are obtained at two base temperatures, $T_{w, 0}=61$ and $81^{\circ} \mathrm{C}$, and two fin lengths, $\mathrm{L}=15$ and $25 \mathrm{~mm}$. The fin base selected is $250 \mathrm{~mm}$. The numerical results indicate that the convective heat transfer rate from the fin array increases as the fin spacing is increased, reaches a maximum at fin spacing, and then starts decreasing again. Thus Fig. 4 shows the optimum fin spacing at which the heat transfer rate is the highest. The results in Fig. 4 indicate that the optimum fin spacing is a function of the base temperature (Tw,0) and fi length (L) also. Optimum fin spacing is $7.3 \mathrm{~mm}$ for fin length $15 \mathrm{~mm}$ and, it is $12 \mathrm{~mm}$ for fin length $25 \mathrm{~mm}$ at base temperature of $81^{\circ} \mathrm{C}$. Fig.5 shows the contributions of convective heat transfer and radiation heat transfer from the eight -fin array on a fixed base of $250 \mathrm{~mm}$. it is observed that both convective and radiation heat transfers increase with an increase in base temperature. It is observed that the maximum radiation contribution is $13 \%$ of the total heat transfer from fin array. 


\section{CONCLUSIONS}

1. A theoretical model is postulated to tackle the problem of heat transfer from a vertical fin array. According to the model, the fin array is assumed to be formed by joining successive two-fin enclosures. The problem for the case of a two-fin enclosure is theoretically formulated and solved considering heat transfer by natural convection and radiation. The problem for the case of a single fin is also formulated and solved to account the heat loss from end fins in the array.

2. Making use of the numerical results for the two-fin enclosure and those for the single fin, a procedure is outlined to assess the total heat transfer rate, average Nusselt number and effectiveness for a fin array. The effect of the system parameters, such as fin spacing, number of fins, fin height, fin base temperature is studied on the total heat transfer from the fin array.

3. A comparison with the experimental data existing in literature indicates satisfactory agreement.

4. Optimum fin spacing exists, which is a function of the fin length.

\section{REFERENCES}

[1] Starner, K.E., Jr. McManus, (1963), “An experimental investigation of free convection heat transfer from rectangular fin arrays", J. Heat Transfer 85, $273-278$.

[2] Charles D. Jones, Lester. F. Smith. (1970). "optimum arrangement of rectangular fins on horizontal surfaces for free convection heat transfer", ASME Journal of Heat Transfer, 92, 6 - 10.

[3] Manzoor, M., Inham, D.B. and Heggs, P.J., (1983) "The one dimensional analysis of fin assembly heat transfer", ASME J. Heat Transfer, 105, $645=651$.

[4] Rammohan Rao, and Venkateshan, S.P., (1996), "Experimental study of free convection and radiation in horizontal fin arrays", Int. J. Heat Mass Transfer, 39, 779 - 789.

[5] Mobedi, M. and Yuncu, H., (2003), "A three dimensional numerical study on natural convection heat Transfer from short rectangular fin array”, Springer - Verlag Heidelberg, Heat Mass transfer, 39, 267 275.

[6] Yuncu, H. and Anbar, G., (1998), “An experimental investigation on performance of fins on a horizontal base in free convection heat transfer”, Springer - Verlag Heidelberg, Heat Mass Transfer, 33, 507 - 514.

[7] Guvenc, A. and Yuncu, H., (2001), "An experimental investigation on performance of fins on a horizontal base in free convection heat transfer”, Springer - Verlag Heidelberg, Heat Mass Transfer, 37 , $409-416$.

[8] Sane, N.K. and Sukhatme, S.P., (1974), "Natural convection heat transfer from horizontal rectangular fin arrays", $5^{\text {th }}$ International Heat and Mass Transfer conference, (3), $114-118$.

[9] Dharma Rao, V., Naidu, S.V., Govinda Rao, B. and Sharma, K.V., (2006), "Heat transfer from horizontal fin array by natural convection and radiation - A conjugate analysis", Int. J, Heat and Mass transfer. (In Press)

[10] Roache, P.J., (1985), “Computational Fluid Dynamics”, Hermosa, Albuquerque, N.M.

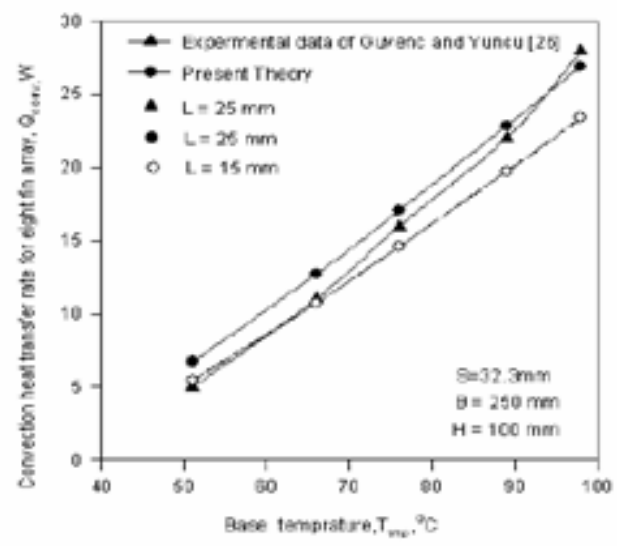

Fig. 2 Comparison of the numerical results with experimental data from literature.

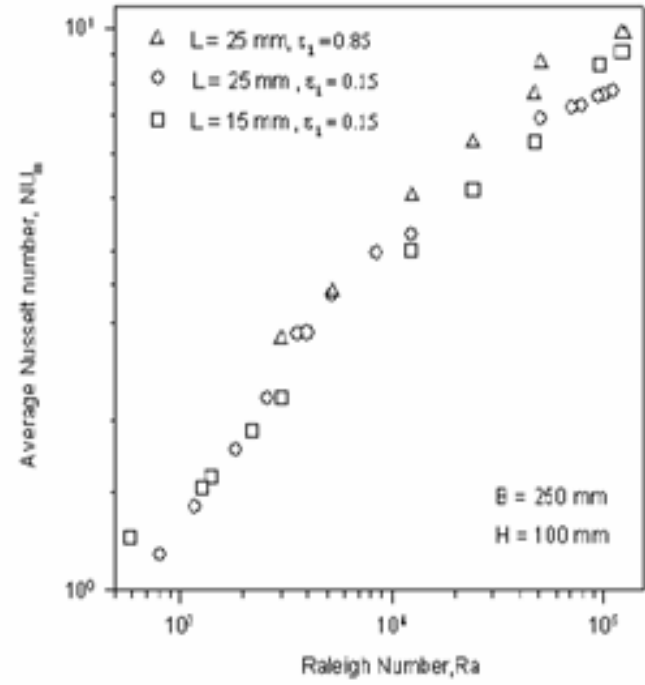

Fig.3 Variation of average Nusselt number with Rayleigh number 


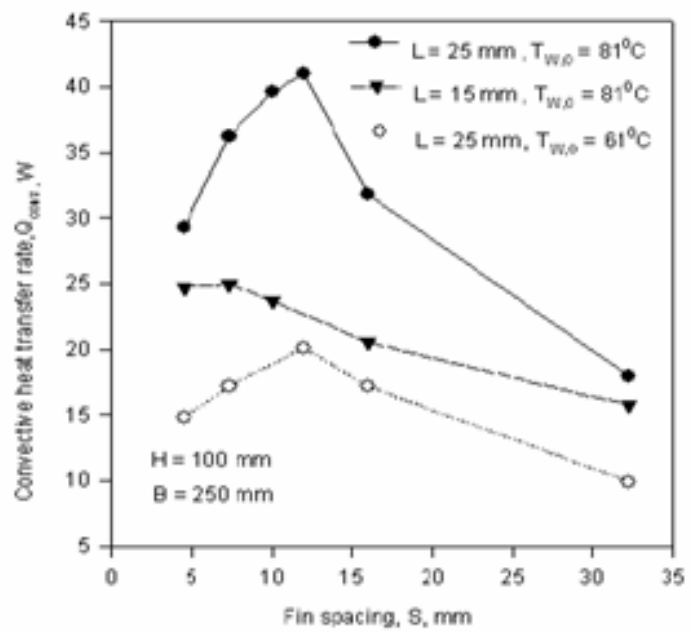

Fig.4. Variation of convective heat transfer rate with fin spacing.

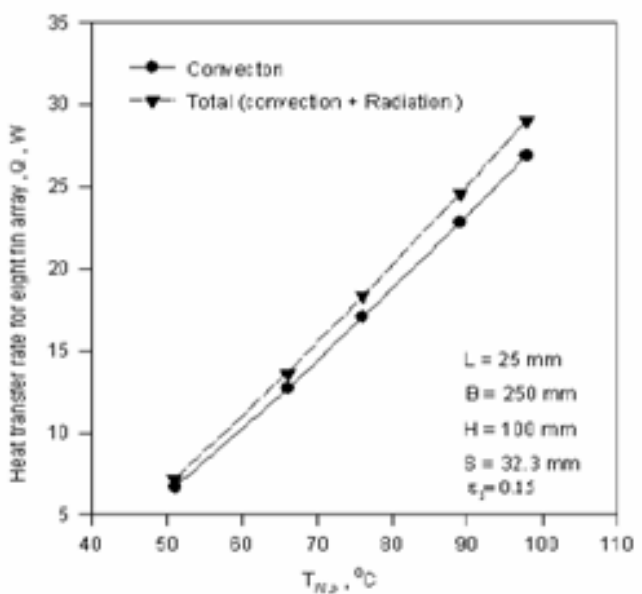

Fig.5. Variation of contributions of convective heat transfer and radiation heat transfer with base temperature. 